\title{
LINEAR PRESERVERS OF POLYNOMIAL NUMERICAL HULLS OF MATRICES
}

\author{
GH. AGHAMOLLAEI ${ }^{1}$, L.W. MARCOUX ${ }^{2 *}$, AND H. RADJAVI $^{3}$
}

\begin{abstract}
Let $\mathbb{M}_{n}$ be the algebra of all $n \times n$ complex matrices, $1 \leq k \leq n-1$ be an integer, and $\varphi: \mathbb{M}_{n} \longrightarrow \mathbb{M}_{n}$ be a linear operator. In this paper, it is shown that $\varphi$ preserves the polynomial numerical hull of order $k$ if and only if there exists a unitary matrix $U \in \mathbb{M}_{n}$ such that either $\varphi(A)=U^{*} A U$ for all $A \in \mathbb{M}_{n}$, or $\varphi(A)=U^{*} A^{t} U$ for all $A \in \mathbb{M}_{n}$.
\end{abstract}

Key words: linear preserver, polynomial numerical hull, numerical range.

AMS Subject Classification 2010: 15A86, 15A60, 15A18.

\section{INTRODUCTION AND PRELIMINARIES}

Let $\mathbb{M}_{n}$ be the algebra of all $n \times n$ complex matrices, and $A \in \mathbb{M}_{n}$. The numerical range, or the field of values, of $A$ is defined as $W(A)=\left\{x^{*} A x: x \in \mathbb{C}^{n}, x^{*} x=1\right\}$. This is useful in studying and understanding both complex matrices and Hilbert space operators, and has many applications in numerical analysis, differential equations, systems theory, etc; (e.g. see [4, 8, and their references). It has been shown (see [1, Lemma 6.22.1]) that

$$
W(A)=\{\lambda \in \mathbb{C}:|\lambda-\mu| \leq\|A-\mu I\|, \quad \forall \mu \in \mathbb{C}\},
$$

where $\|$.$\| is the usual operator norm on \mathbb{M}_{n}$ (i.e., the norm on $\mathbb{M}_{n}$ obtained through its action on $\mathbb{C}^{n}$, where $\mathbb{C}^{n}$ carries the usual Euclidean norm), and $I$ is the $n \times n$ identity matrix. Now, let $k$ be a positive integer and denote by $\mathbb{P}_{k}$ the set of all scalar polynomials of degree $k$ or less. Using the formulation of $W(A)$ given in (1), the concept of numerical range of $A$ has been generalized to that of the polynomial numerical hull of order $k$ of $A$, which is defined and denoted (e.g., see [12]) by

$$
V^{k}(A)=\left\{\lambda \in \mathbb{C}:|p(\lambda)| \leq\|p(A)\| \text { for all } p \in \mathbb{P}_{k}\right\}
$$

This is a set designed to give more information than the spectrum and numerical range alone can provide about the behaviour of the matrix $A$ under the action of polynomials and other functions

Date: March 25, 2019.

${ }^{1}$ Research supported in part by Shahid Bahonar University of Kerman, Kerman, Iran.

${ }^{2}$ Research supported in part by NSERC (Canada), ${ }^{*}$ Corresponding author. 
of $A$, and has many applications in the study of convergence of iterative methods in solving linear systems. For more information, we refer the reader to [6] and [7] and their references (see also [12]).

In the following proposition, we state some properties of polynomial numerical hulls of matrices which will be useful in our discussion. For proofs of these and a number of other properties, we suggest [3] and [6].

Proposition 1.1. Let $A \in \mathbb{M}_{n}$. Then the following assertions are true:

(i) $V^{k}(A)$ is a compact set in $\mathbb{C}$;

(ii) $\sigma(A)=V^{m}(A) \subseteq \cdots \subseteq V^{k+1}(A) \subseteq V^{k}(A) \subseteq \cdots \subseteq V^{1}(A)=W(A)$, where $m \geq n$;

(iii) $V^{k}(\alpha A+\beta I)=\alpha V^{k}(A)+\beta$, where $\alpha, \beta \in \mathbb{C}$;

(iv) $V^{k}\left(U^{*} A U\right)=V^{k}(A)$, where $U \in \mathbb{M}_{n}$ is unitary;

(v) $V^{k}\left(A^{T}\right)=V^{k}(A)$ and $V^{k}(\bar{A})=\overline{V^{k}(A)}:=\left\{\bar{\lambda}: \lambda \in V^{k}(A)\right\}$. Consequently, $V^{k}\left(A^{*}\right)=\overline{V^{k}(A)}$;

(vi) $V^{k}(A)=\left\{\lambda \in \mathbb{C}:\left(\lambda, \lambda^{2}, \ldots, \lambda^{k}\right) \in \operatorname{conv}\left(W\left(A, A^{2}, \ldots, A^{k}\right)\right)\right\}$, where conv $(\cdot)$ denotes the convex hull, and $W\left(A_{1}, A_{2}, \ldots, A_{k}\right):=\left\{\left(x^{*} A_{1} x, x^{*} A_{2} x, \ldots, x^{*} A_{k} x\right): x \in \mathbb{C}^{n}, x^{*} x=1\right\}$ is the joint numerical range of $\left(A_{1}, A_{2}, \ldots, A_{k}\right) \in \mathbb{M}_{n}^{k}$;

(vii) If $A$ is Hermitian, then $V^{k}(A)= \begin{cases}\operatorname{conv}(\sigma(A)) & \text { for } k=1, \\ \sigma(A) & \text { for } k \geq 2 .\end{cases}$

Regarding the polynomial numerical hulls of Jordan blocks, we have the following result which can be found in [5, Section 2].

Proposition 1.2. Let $J$ be an $n \times n$ Jordan block with eigenvalue 0 . If $k=1,2, \ldots, n-1$, then $V^{k}(J)$ is a circular disk about the origin of radius $0<r_{k}<1$, and for $k \geq n, V^{k}(J)=\{0\}$.

An active and popular research area in matrix and operator theory is the study of linear preserver problems. Typically, one attempts to classify those linear maps $\varphi: \mathbb{M}_{n} \rightarrow \mathbb{M}_{n}$ which preserve some property of matrices (such as the rank, or the spectrum, etc.). Examples of such problems can be found in [10]. The purpose of this paper is to characterize linear operators preserving the polynomial numerical hull of order $k$ of matrices. Let $k \geq n$, and $\varphi: \mathbb{M}_{n} \longrightarrow \mathbb{M}_{n}$ be a linear operator satisfying $V^{k}(\varphi(A))=V^{k}(A)$ for all $A \in \mathbb{M}_{n}$. In this case, for every $A \in \mathbb{M}_{n}, V^{k}(A)$ reduces to $\sigma(A)$, and then, by [11, Theorem 3], there exists a nonsingular matrix $S \in \mathbb{M}_{n}$ such that either $\varphi(A)=S^{-1} A S$ for all $A \in \mathbb{M}_{n}$, or $\varphi(A)=S^{-1} A^{t} S$ for all $A \in \mathbb{M}_{n}$. This reduces our problem to the case where $1 \leq k \leq n-1$, and we characterize linear preservers of $V^{k}(\cdot)$ on $\mathbb{M}_{n}$ in Section 2 of this paper (see Theorem 2.5 below). 


\section{MAIN RESULTS}

Let $k \in \mathbb{N}$. A linear operator $\varphi: \mathbb{M}_{n} \longrightarrow \mathbb{M}_{n}$ is called a linear preserver of the polynomial numerical hull of order $k$ if $V^{k}(\varphi(A))=V^{k}(A)$ for all $A \in \mathbb{M}_{n}$. Our first goal is to show that $\varphi$ is a unital bijective map. For this, we require the following lemma.

Lemma 2.1. Let $A \in \mathbb{M}_{n}, \alpha \in \mathbb{C}$, and $k \in \mathbb{N}$. Then

$$
V^{k}(A+X)=V^{k}(X)+\alpha \text { for all } X \in \mathbb{M}_{n} \Longleftrightarrow A=\alpha I
$$

Proof. In view of Proposition 1.1 (iii), without loss of generality, we assume that $\alpha=0$.

Now, suppose that $V^{k}(A+X)=V^{k}(X)$ for all $X \in \mathbb{M}_{n}$. We will show that $A=0$. By setting $X=0$, we see that $\sigma(A) \subseteq V^{k}(A)=\{0\}$. Moreover, $\sigma\left(A+A^{*}\right) \subseteq V^{k}\left(A+A^{*}\right)=V^{k}\left(A^{*}\right)=\{0\}$ (by Proposition 1.1 above). Since $A+A^{*}$ is hermitian, we conclude that $A=-A^{*}$, so that $A$ is normal. But $A$ normal and $\sigma(A)=\{0\}$ implies that $A=0$, as required.

The converse is a trivial consequence of Proposition 1.1 (iii). This completes the proof.

Lemma 2.2. Let $k \in \mathbb{N}$, and $\varphi: \mathbb{M}_{n} \longrightarrow \mathbb{M}_{n}$ be a linear operator preserving the polynomial numerical hull of order $k$. Then $\varphi$ is bijective and $\varphi(I)=I$.

Proof. To prove that $\varphi$ is bijective, we suppose, for $A \in \mathbb{M}_{n}$, that $\varphi(A)=0$, and we will show that $A=0$. In view of Lemma 2.1, it is enough to show that for every $X \in \mathbb{M}_{n}, V^{k}(A+X)=V^{k}(X)$.

Let $X \in \mathbb{M}_{n}$ be given. Then, by our assumption, we have that

$$
V^{k}(A+X)=V^{k}(\varphi(A+X))=V^{k}(\varphi(A)+\varphi(X))=V^{k}(\varphi(X))=V^{k}(X) .
$$

To prove the second assertion, we show that $\varphi(I)-I=0$. Again, using Lemma 2.1, it suffices to show that given $X \in \mathbb{M}_{n}$ arbitrary, we have $V^{k}((\varphi(I)-I)+X)=V^{k}(X)$. Since $\varphi$ is surjective, there exists $C \in \mathbb{M}_{n}$ such that $\varphi(C)=X$. Now, by Proposition 1.1 (iii), we have

$$
V^{k}((\varphi(I)-I)+X)=V^{k}(\varphi(I)+X)-1=V^{k}(\varphi(I+C))-1=V^{k}(C)=V^{k}(\varphi(C))=V^{k}(X) .
$$

This completes the proof.

We shall require the following notation. We denote by $\mathcal{U}_{n}:=\left\{U \in \mathbb{M}_{n}: U^{*} U=I\right\}$ the group of all $n \times n$ unitary matrices, by $G L_{n}(\mathbb{C}):=\left\{A \in \mathbb{M}_{n}: \operatorname{det}(A) \neq 0\right\}$ the general linear group of $n \times n$ nonsingular complex matrices, and by $\mathbb{C}^{*}$ the set of all non-zero complex numbers. Given $\gamma \in \mathbb{C}^{*}$, we denote by $\widehat{\gamma}$ the vector $\left(1, \gamma, \gamma^{2}, \ldots, \gamma^{n-1}\right) \in\left(\mathbb{C}^{*}\right)^{n}$. We also write $\mathfrak{S}_{n}$ to denote the symmetric group of all permutations of $\{1,2, \ldots, n\}$. Given a vector $d=\left(d_{1}, d_{2}, \ldots, d_{n}\right) \in \mathbb{C}^{n}$, we 
write $D_{d}=\operatorname{diag}\left(d_{1}, d_{2}, \ldots, d_{n}\right)$, and given $\sigma \in \mathfrak{S}_{n}$, we shall denote by $D_{d}^{\sigma}$ the diagonal matrix $D_{d}^{\sigma}=\operatorname{diag}\left(d_{\sigma(1)}, d_{\sigma(2)}, \ldots, d_{\sigma(n)}\right)$. Finally, let us also introduce the sets:

$$
\mathcal{G}_{k}:=\left\{X \in G L_{n}(\mathbb{C}): V^{k}\left(X^{-1} A X\right)=V^{k}(A) \text { for all } A \in \mathbb{M}_{n}\right\}
$$

where $k \in \mathbb{N}$, and

$$
\mathbb{C}^{*} \mathcal{U}_{n}:=\left\{\alpha U: \alpha \in \mathbb{C}^{*}, U \in \mathcal{U}_{n}\right\}
$$

It is easy to see that $\mathcal{G}_{k}$ and $\mathbb{C}^{*} \mathcal{U}_{n}$ are subgroups of $G L_{n}(\mathbb{C})$, and by Proposition $1.1(i v), \mathbb{C}^{*} \mathcal{U}_{n} \subseteq \mathcal{G}_{k}$. In fact, we have the following result.

Lemma 2.3. Let $n, k$ be two positive integers such that $n \geq 2$ and $k \leq n-1$. Moreover, let $\mathcal{G}_{k}$ and $\mathbb{C}^{*} \mathcal{U}_{n}$ be the groups defined as in (2) and (3), respectively. Then $\mathcal{G}_{k}=\mathbb{C}^{*} \mathcal{U}_{n}$.

Proof. It is enough to show that $\mathcal{G}_{k} \subseteq \mathbb{C}^{*} \mathcal{U}_{n}$. Suppose that $S \in \mathcal{G}_{k}$, and that $S \notin \mathbb{C}^{*} \mathcal{U}_{n}$. Let $S=U|S|$ be the polar decomposition of $S$. Note that $U \in \mathcal{U}_{n}$ and $|S|=\left(S^{*} S\right)^{\frac{1}{2}}$. Since $\mathcal{U}_{n} \subseteq \mathcal{G}_{k},|S|=U^{*} S$, and $\mathcal{G}_{k}$ is a group, $|S| \in \mathcal{G}_{k}$, and without loss of generality, we may assume that $|S|=\operatorname{diag}\left(s_{1}, s_{2}, \ldots, s_{n}\right)$, where $0<s_{1} \leq s_{2} \leq \cdots \leq s_{n}$. Moreover, since $S \notin \mathbb{C}^{*} \mathcal{U}_{n}$, it follows that $|S| \neq \alpha I$ for all $\alpha \in \mathbb{C}^{*}$, and thus $s_{n}>s_{1}$. Furthermore, since $s_{1}^{-1} I \in \mathcal{G}_{k}$ and the latter is a group, it follows that $D:=s_{1}^{-1}|S| \in \mathcal{G}_{k}$. Let $r_{i}=s_{1}^{-1} s_{i}$ for $i=2,3, \ldots, n, r=\left(1, r_{2}, r_{3}, \ldots, r_{n}\right)$, so that $D_{r}=\operatorname{diag}\left(1, r_{2}, r_{3}, \ldots, r_{n}\right)$. Consider the permutation $\sigma \in \mathfrak{S}_{n}$ defined by:

$$
\sigma(i)= \begin{cases}1 & \text { if } i=1 \\ i+1 & \text { if } 2 \leq i<n \\ 2 & \text { if } i=n\end{cases}
$$

Then $D_{r}^{\sigma^{j}}$ is unitarily equivalent to $D$. Since $D \in \mathcal{G}_{k}$ and $\mathcal{G}_{k}$ is a group containing all unitary matrices, it follows that $D_{r}^{\sigma^{j}} \in \mathcal{G}_{k}$ for all $j \geq 1$, and also

$$
M:=D \cdot D_{r}^{\sigma} \cdot D_{r}^{\sigma^{2}} \cdots D_{r}^{\sigma^{n-1}} \in \mathcal{G}_{k} .
$$

Observe that

$$
M=\operatorname{diag}(1, \alpha, \alpha, \ldots, \alpha) \in \mathbb{M}_{n},
$$

where $\alpha=r_{2} r_{3} \cdots r_{n}>1$. Again, the fact that $\mathcal{G}_{k}$ is a group containing $\mathbb{C}^{*} I$ implies that

$$
P:=\alpha M^{-1}=\operatorname{diag}(\alpha, 1,1, \ldots, 1) \in \mathcal{G}_{k} .
$$


By setting $P_{j}:=\operatorname{diag}(1,1, \ldots, 1, \alpha, 1,1, \ldots, 1) \in \mathcal{G}_{k}$, where $j=2,3, \ldots, n-1$, and the unique $\alpha$ appears in the $j^{\text {th }}$ coordinate, we see that $P_{j}$ is unitarily equivalent to $P$, and so $P_{j} \in \mathcal{G}_{k}$. Also, observe that again, as $\mathcal{G}_{k}$ is a group, we have

$$
D_{\widehat{\alpha}}=P_{2} P_{3}^{2} P_{4}^{3} \cdots P_{n}^{n-1} \in \mathcal{G}_{k} .
$$

This implies that

$$
V^{k}\left(D_{\widehat{\alpha}}^{-1} J D_{\widehat{\alpha}}\right)=V^{k}(J),
$$

where $J$ is the $n \times n$ Jordan block with eigenvalue 0. By Proposition 1.2 and the fact that $k<n$, there exists $\rho>0$ such that $V^{k}(J)=\{z \in \mathbb{C}:|z| \leq \rho\}$. Observe that $D_{\widehat{\alpha}}^{-1} J D_{\widehat{\alpha}}=\alpha J$, and so, by Proposition 1.1 (iii),

$$
V^{k}(\alpha J)=\alpha V^{k}(J)=\{z \in \mathbb{C}:|z| \leq \alpha \rho\}
$$

which contradicts (4) because $\alpha>1$. Thus $\mathcal{G}_{k} \subseteq \mathbb{C}^{*} \mathcal{U}_{n}$, and so the proof is complete.

To reach our goal, we also need the following lemma.

Lemma 2.4. Let $2 \leq k \in \mathbb{N}$, and $\varphi: \mathbb{M}_{n} \longrightarrow \mathbb{M}_{n}$ be a linear operator preserving the polynomial numerical hull of order $k$. Then $\operatorname{tr}(\varphi(H))=\operatorname{tr}(H)$ for all Hermitian matrices $H \in \mathbb{M}_{n}$.

Proof. Consider the following two steps:

Step 1: Let $P$ and $Q$ be two nonzero rank-one orthogonal projections in $\mathbb{M}_{n}$ such that $P Q=Q P=$ 0 . Then $\operatorname{tr}(\varphi(P))=\operatorname{tr}(\varphi(Q))$.

To see this, observe that by our assumptions on $P$ and $Q$, we have $\sigma(P)=\sigma(Q)=\{0,1\}$, and clearly there exists a unitary matrix $U \in \mathbb{M}_{n}$ such that $U^{*} P U=Q$. Since $\mathcal{U}_{n}$ is a connected set in $\mathbb{M}_{n}$, there exists a continuous path $\left\{U_{t}: 0 \leq t \leq 1\right\}$ of unitary matrices in $\mathbb{M}_{n}$ such that $U_{0}=I$ and $U_{1}=U$. Since $k \geq 2$, Proposition 1.1 ( (ii) and (vii)) and our assumption on $\varphi$ show that for every $0 \leq t \leq 1, \sigma\left(\varphi\left(P_{t}\right)\right) \subseteq V^{k}\left(\varphi\left(P_{t}\right)\right)=V^{k}\left(P_{t}\right)=\sigma\left(P_{t}\right)=\{0,1\}$, where $P_{t}=U_{t}^{*} P U_{t}$. By the continuity of $\varphi$, we find that $\sigma\left(\varphi\left(P_{t}\right)\right)=\sigma\left(\varphi\left(P_{0}\right)\right)$, counting multiplicities, for all $0 \leq t \leq 1$. Consequently, $\sigma(\varphi(P))=\sigma(\varphi(Q))$, counting multiplicities, and hence, $\operatorname{tr}(\varphi(P))=\operatorname{tr}(\varphi(Q))$.

Step 2: Let $P$ be a nonzero rank-one orthogonal projection in $\mathbb{M}_{n}$. Then $\operatorname{tr}(\varphi(P))=1$.

To prove the assertion in Step 2 , let $\left\{x_{1}, x_{2}, \ldots, x_{n}\right\}$ be an orthonormal basis for $\mathbb{C}^{n}$ such that $P=$ $x_{1}^{*} x_{1}$. By setting $P_{1}=P=x_{1}^{*} x_{1}, P_{2}=x_{2}^{*} x_{2}, \ldots, P_{n}=x_{n}^{*} x_{n}$, we see that $P_{1}, P_{2}, \ldots, P_{n}$ are nonzero rank-one orthogonal projections such that $P_{i} P_{j}=P_{j} P_{i}=0$ for every $i \neq j$, and $P_{1}+P_{2}+\cdots+P_{n}=I$. 
Now by Lemma 2.2 and Step 1, we have

$$
n=\operatorname{tr}(I)=\operatorname{tr}(\varphi(I))=\operatorname{tr}\left(\varphi\left(\sum_{i=1}^{n} P_{i}\right)\right)=\sum_{i=1}^{n} \operatorname{tr}\left(\varphi\left(P_{i}\right)\right)=n \operatorname{tr}(\varphi(P)) .
$$

This shows that $\operatorname{tr}(\varphi(P))=1$.

Next, let $H \in \mathbb{M}_{n}$ be a Hermitian matrix. Then there exist real numbers $d_{1}, d_{2}, \ldots, d_{n}$ and nonzero rank-one orthogonal projections $P_{1}, P_{2}, \ldots, P_{n}$ such that $H=\sum_{i=1}^{n} d_{i} P_{i}$. By Step 2, we have

$$
\operatorname{tr}(\varphi(H))=\sum_{i=1}^{n} d_{i} \operatorname{tr}\left(\varphi\left(P_{i}\right)\right)=\sum_{i=1}^{n} d_{i}=\operatorname{tr}(H)
$$

This completes the proof.

We are now ready to characterize the linear preservers of polynomial numerical hulls of matrices.

Theorem 2.5. Let $n, k$ be two positive integers, $n \geq 2$ and $k \leq n-1$. Moreover, let $\varphi: \mathbb{M}_{n} \longrightarrow \mathbb{M}_{n}$ be a linear operator. Then $V^{k}(\varphi(A))=V^{k}(A)$ for all $A \in \mathbb{M}_{n}$ if and only if there exists a unitary matrix $U \in \mathbb{M}_{n}$ such that either $\varphi(A)=U^{*} A U$ for all $A \in \mathbb{M}_{n}$, or $\varphi(A)=U^{*} A^{t} U$ for all $A \in \mathbb{M}_{n}$.

Proof. The assertion for the cases where $k=1$ or $n=2$ follow from [9, Theorem 3] and the fact that $V^{1}(\cdot)$ coincides with the numerical range. As such, we may assume that $n \geq 3$ and $2 \leq k \leq n-1$.

Let $H \in \mathbb{M}_{n}$ be an arbitrary Hermitian matrix. By Proposition 1.1 ( (ii) and (vii)) and our hypotheses, we have

$$
\sigma(\varphi(H)) \subseteq V^{k}(\varphi(H))=V^{k}(H)=\sigma(H) .
$$

Using Lemma 2.4, we may argue in the same manner as in the proof of Lemma 3 of [2, p. 2677] to deduce that $\sigma(\varphi(H))=\sigma(H)$ for any arbitrary Hermitian matrix $H \in \mathbb{M}_{n}$. So, by [11, Theorem 3], there exists a nonsingular matrix $S \in G L_{n}(\mathbb{C})$ such that either $\varphi(A)=S^{-1} A S$ for all $A \in \mathbb{M}_{n}$, or $\varphi(A)=S^{-1} A^{t} S$ for all $A \in \mathbb{M}_{n}$.

Suppose, as a first case, that $\varphi(A)=S^{-1} A S$ for all $A \in \mathbb{M}_{n}$. Since $V^{k}(\varphi(A))=V^{k}(A)$ for all $A \in \mathbb{M}_{n}, S \in \mathcal{G}_{k}$, where $\mathcal{G}_{k}$ is the group defined in (2) above. Since $k<n$, Lemma 2.3 implies that $S \in \mathbb{C}^{*} \mathcal{U}_{n}$, where $\mathbb{C}^{*} \mathcal{U}_{n}$ is the group as in $(3)$, and so, there exist $\alpha \in \mathbb{C}^{*}$ and a unitary matrix $U \in \mathcal{U}_{n}$ such that $S=\alpha U$. Therefore, for every $A \in \mathbb{M}_{n}, \varphi(A)=S^{-1} A S=U^{*} A U$, and so the result holds.

The result in the second case, i.e., $\varphi(A)=S^{-1} A^{t} S$ for all $A \in \mathbb{M}_{n}$, follows from Proposition $1.1(v)$ and an argument similar to that used in the proof of the first case above.

Finally, the converse of the assertion follows easily from Proposition $1.1((i v)$ and $(v))$, completing the proof. 
Acknowledgement. This paper was written when the first author was spending his sabbatical leave (July 2018-July 2019) at the Department of Pure Mathematics, University of Waterloo, Waterloo, Ontario, Canada. He thanks the staff and faculty members of the department for their kind support.

The authors wish to thank the anonymous referee for his/her helpful comments.

\section{REFERENCES}

[1] F.F. Bonsall and J. Duncan, Numerical Ranges II, London Mathematical Society Lecture Notes Series, Cambridge University Press, New York, 1973.

[2] C. Costara, Maps on matrices that preserve the spectrum, Linear Algebra Appl. 435 (2011), 2674-2680.

[3] Ch. Davis, C.K. Li and A. Salemi, Polynomial numerical hulls of matrices, Linear Algebra Appl. 428 (2008), 137-153.

[4] M. Eiermann, Field of values and iterative methods, Linear Algebra Appl. 180 (1993), 167-197.

[5] V. Faber, A. Greenbaum and D.E. Marshall, The polynomial numerical hulls of Jordan blocks and related matrices, Linear Algebra Appl. 374 (2003), 231-246.

[6] A. Greenbaum, Generalizations of the field of values useful in the study of polynomial functions of a matrix, Linear Algebra Appl. 347 (2002), 233-249.

[7] A. Greenbaum, Upper and lower bounds on norms of functions of matrices, Linear Algebra Appl. 430 (2009), 52-65.

[8] R. Horn and C. Johnson, Topics in Matrix Analysis, Cambridge University Press, New York, 1991.

[9] C. K. Li, Linear operators preserving the numerical radius of matrices, Proc. Amer. Math. Soc.. 99 (1987), 601-608.

[10] C.K. Li and S. Pierce, Linear preserver problems, The American Math. Monthly, 108 (2001), 591-605.

[11] M. Marcus and B.N. Moyls, Linear transformations on algebras of matrices, Canad. J. Math. 11 (1959), 61-66.

[12] O. Nevanlinna, Convergence of iterations for linear equations, Birkhäuser, Basel, 1993. 
Department of Pure Mathematics, Faculty of Mathematics and Computer, Shahid Bahonar University of Kerman, Kerman, Iran

Mahani Mathematical Research Center, Shahid Bahonar University of Kerman, KERMAN, IRAN

E-mail address: aghamollaei@uk.ac.ir, aghamollaei1976@gmail.com

Department of Pure Mathematics, University of Waterloo, Waterloo, Ontario, Canada N2L 3G1

E-mail address: Laurent.Marcoux@uwaterloo.ca

Department of Pure Mathematics, University of Waterloo, Waterloo, Ontario, CANADA N2L 3G1

E-mail address: HRadjavi@uwaterloo.ca 